\title{
Subclavian Fat Biopsy with the Pacemaker Implantation is Useful for the Diagnosis of Amyloidosis
}

\author{
Keiji Matsunaga, Yusuke Hasui,Kaori Ishikawa,Kazushi Murakami, Takahisa Noma and Tetsuo \\ Minamino*
}

Department of Cardiorenal and Cerebrovascular Medicine, Kagawa University, Japan

Received: May 31, 2018; Published: June 14, 2018

*Corresponding author: Tetsuo Minamino, Department of Cardiorenal and Cerebrovascular Medicine, Japan

\begin{abstract}
Amyloidosis has been regarded as a rare disease, however, recent reports demonstrated that the prevalence of wild type ATTR amyloidosis is up to $25 \%$ in elderly persons aged 80 years or older. Progressive conduction disease is common in ATTR amyloidosis and approximately 20 $40 \%$ of patients with amyloidosis is estimated to require the pacemaker implantation. However, the diagnosis of amyloidosis in these patients would be underestimated because the invasive biopsy for the diagnosis is often hesitated in elderly persons. Therefore, it is ideal to perform the invasive biopsy to diagnose amyloidosis at the timing of pacemaker implantation. We here report the case that the subclavian fat biopsy with the pacemaker implantation was useful for the diagnosis of amyloidosis.
\end{abstract}

Keywords: Amyloidosis; Cardiac amyloidosis; Subclavian Fat Biopsy; Pacemaker Implant

\section{Introduction}

Amyloidosis has been regarded as a rare disease, however, recent reports demonstrated that the prevalence of wild type ATTR amyloidosis (ATTRwt) is up to $25 \%$ in elderly persons aged 80 years or older[1].Progressive conduction disease is common in ATTR amyloidosis and approximately 20-40\% of patients with amyloidosis is estimated to require the pacemaker implantation. Because many promising diseasemodifying therapies have emerged over the past decade[2]the correct diagnosis of amyloidosis will become more important for better treatment. However, the diagnosis of amyloidosis in these patients would beunderestimated because the additional invasive biopsy is often hesitated in elderly persons.Therefore, it is ideal to perform the invasive biopsy to diagnose amyloidosis at the timing of pacemaker implantation. We here report the case that the subclavian fat biopsy with the pacemaker implantation was useful for the diagnosis of amyloidosis.

\section{Case Report}

A 82yearold man with heart failure due to bradycardia was referred to our hospital. Systolic blood pressure was $71 \mathrm{mmHg}$ and pulse rate was $32 \mathrm{beats} / \mathrm{min}$ with cold sweat. Electrocardiogram(ECG) showed sinus bradycardia with low voltage in limb leads and pseudo infarct pattern in precordial leads(Figure 1).A chest X-ray showed cardiac dilatation with bilateral pleuraleffusion, and echocardiographicevaluation demonstrated a reduced left ventricular ejection fraction (29\%) with cardiac hypertrophy (interventricular septal septum $17 \mathrm{~mm}$ and left ventricular posterior wall thickness $18 \mathrm{~mm}$ ).We diagnosed that cardiogenic shock occurred due to bradycardia. We inserted temporary transvenous cardiac pacing to keep circulatory dynamics and implanted a permanent dual chamber pacemaker electively. Since clinical features suggested us that he was cardiac amyloidosis, we recommended the cardiac biopsy to the patient. However,the patient did not agree with the cardiac biopsy,we performed the subclavian fat biopsy with the pacemaker implantation and obtained the specimen ( $5 \mathrm{~mm} \times 5 \mathrm{~mm})$. Pathological tests showed Congo Red stain positive (Figures2-4),so he was diagnosed as ATTRwt with clinical findings. He had given a drug adjustment based on the diagnostic result and left the hospital without symptoms due to heart failure.

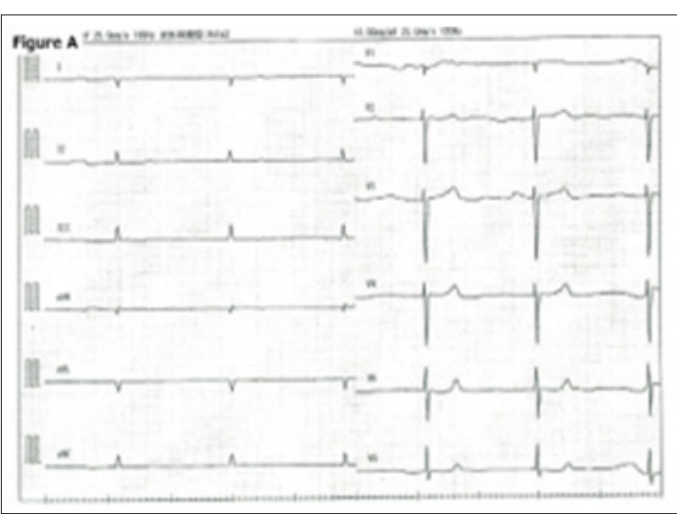

Figure1: Electrocardiogram at admission. 


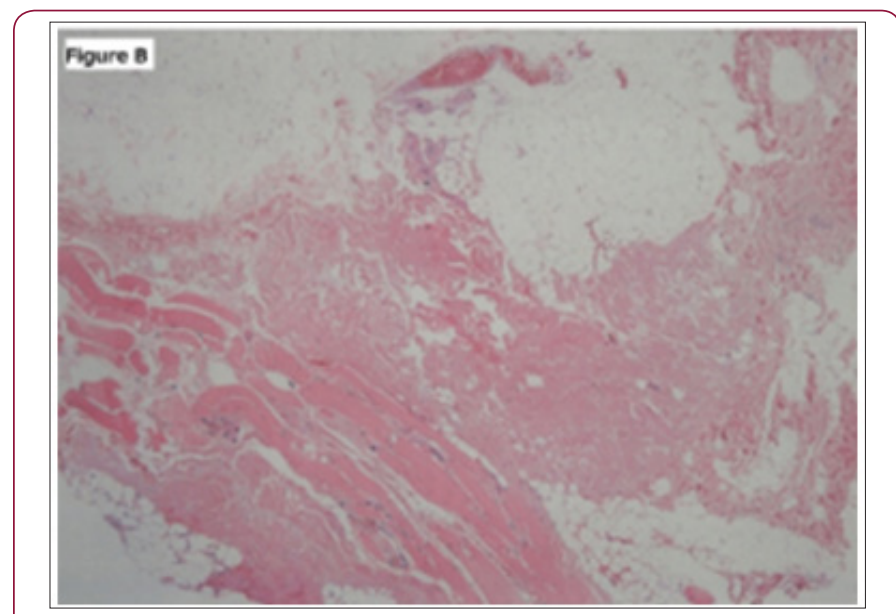

Figure 2: Subclavian fat tissue. Hematoxyline-Eosin (X40).

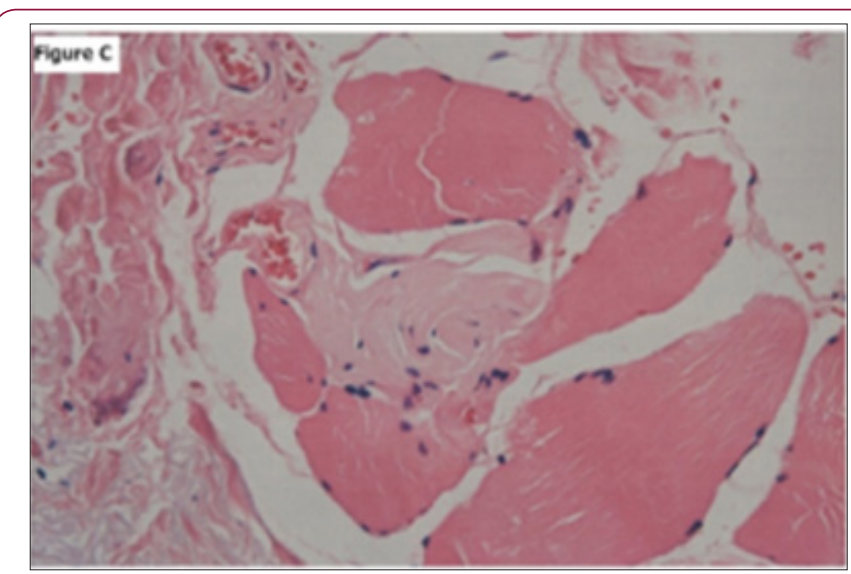

Figure 3: Subclavian fat tissue. Hematoxyline-Eosin (X400).

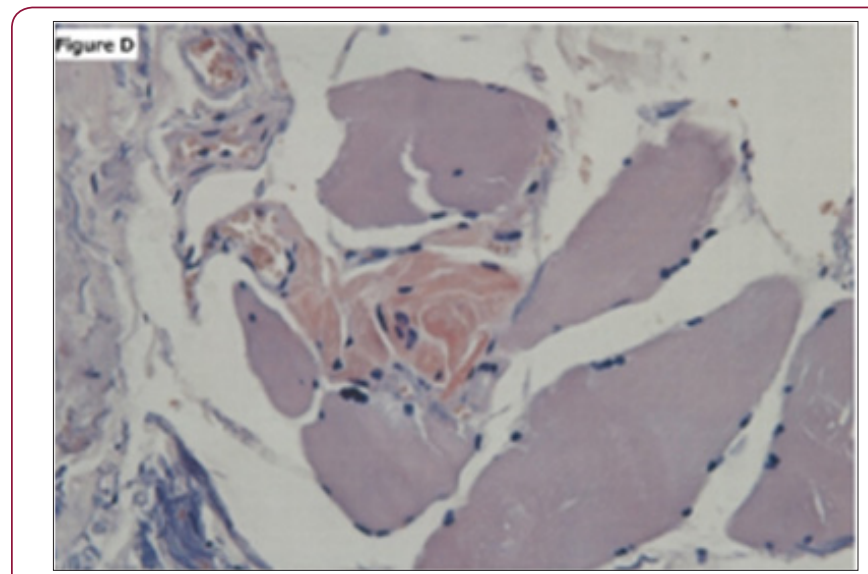

Figure 4: Subclavian fat tissue. Congo red (X400).

\section{Discussion}

We report a case of amyloidosis who was diagnosed by subclavian fat biopsy with the pacemakerimplantation. We have learned 2 important issues from the case reported here.First, the case of amyloidosis who needs pacemaker implantation is increasingly recognized.Recent reports demonstrated that the prevalence of ATTRwt is up to $25 \%$ in elderly persons aged 80 years or older,assuming that approximately $20-40 \%$ of patients with amyloidosis is estimated to require the pacemaker implantation[2] and that the patients who need pacemaker implantation due to amyloidosis are estimated about $5-10 \%$ in the total population aged 80 years or older.

These things strongly suggest that patients who need pacemaker implantation will include many undiagnosed amyloidosis patients. Because the drugs for cardiac amyloidosis have been intensively developed over the past decades, we need correct diagnosis for the better treatment ofamyloidosis in the future.Second, subclavian fat biopsy with the pacemaker implantation is useful for the diagnosis of amyloidosis. Abdominal fat aspiration is noninvasive and popular methods, but its diagnostic sensitivity is only $15 \%$ in the case of ATTRwt[3]. On the other hands, in the article of Ikeda et al, skin biopsy from the abdominal wall, which can get the deep layer of subcutaneous fat tissue, showed $73 \%$ sensitivity in the case of ATTRwt[4]. Biopsy from the subclavian fat at the pacemaker implantation can get the deep layer of subcutaneous fat tissue,it is expected that subclavian fat biopsy would show equivalent sensitivity compared with the biopsy from the abdominal wall. Although the diagnosis from the subclavian fat biopsy is limited, we always need to consider the performance of the subclavian fat biopsy due to its high feasibility without additional invasion. In the future, we can routinely perform this biopsy to diagnosis many hidden amyloidosis, increasing the opportunity for the better treatment with specific drug for amyloidosis.

\section{Conclusion}

The subclavian fat biopsy with the pacemaker implantation can be performed without additional intervention. When we can't deny the possibility of amyloidosis in the case of pacemaker implantation, we should always consider the subclavian fat biopsy.

\section{References}

1. Julian D Gillmore, Mathew S Maurer, Rodney H Falk, Giampaolo Merlini, Thibaud Damy, et al. (2016) Nonbiopsy Diagnosis of Cardiac Transthyretin Amyloidosis. Circulation 133(24): 2404-2412.

2. Castano A, Drachman BM, Judge D, Maurer MS (2015) Natural history and therapy of TTR-cardiac amyloidosis: emerging disease modifying therapies from organ transplantation to stabilizer and silencer drugs. Heart failure reviews 20(2): 163-178.

3. Quarta CC, Gonzalez Lopez E, Gilbertson JA, Botcher N, Rowczenio D, et al. (2017) Diagnostic sensitivity of abdominal fat aspiration in cardiac amyloidosis. Eur Heart J 38(24): 1905-1908.

4. Ikeda SI, Sekijima Y, Tojo K, Koyama J (2011) Diagnostic value of abdominal wall fat pad biopsy in senile systemic amyloidosis. Amyloid 18(4): 211-215. 
(C) (i) This work is licensed under Creative Submission Link: https://biomedres.us/submit-manuscript.php

\begin{tabular}{ll} 
BIOMEDICAL & Assets of Publishing with us \\
RESEARCHES & - Global archiving of articles \\
& - Immediate, unrestricted online access \\
& - Rigorous Peer Review Process \\
\hline https://biomedres.us/
\end{tabular}

